\title{
Half Inverse Problem for the Sturm-Liouville Operator with Coulomb potential
}

\author{
Murat Sat* \\ Departments of Mathematics, Erzincan University, Faculty of Science and Art, 24100, Erzincan, Turkey
}

Received: 11 Mar. 2013, Revised: 11 Jul. 2013, Accepted: 13 Jul. 2013

Published online: 1 Mar. 2014

\begin{abstract}
In this paper, we discuss a half inverse problem for the Sturm-Liouville operator with Coulomb potential and show that if $\mathrm{q}(\mathrm{x})$ is prescribed on $\left[\frac{\pi}{2}, \pi\right]$ then only one spectrum is sufficient to determine potential function $\mathrm{q}(\mathrm{x})$ on the whole interval $(0, \pi]$.
\end{abstract}

Keywords: Half inverse problem, eigenvalue, Coulomb potential

\section{Introduction}

The theory of inverse problems for differential operators occupies an important position in the current development of the spectral theory of linear operators. Inverse problems of spectral analysis consist in the recovery of operators from their spectral data $[1-6]$. Half inverse problem for a Sturm-Liouville operator consists in reconstruction of this operator from its spectrum and half of the potential.

The first result on the half inverse problem is due to Hochstadt and Lieberman [7], who proved that if $\left\{\lambda_{n}\right\}=\left\{\widetilde{\lambda}_{n}\right\}, q=\widetilde{q}, H=\widetilde{H}$, on $\left(\frac{1}{2}, 1\right)$ then $q=\widetilde{q}$ on $(0,1)$. Later Hald [8] showed that if the potential is known over half the interval and if one boundary condition is given then the potential and the other boundary condition are uniquely determined by the eigenvalues. The position of the discontinuity and the jump in the eigenfunction is also uniquely determined. This is a generalized theorem by Hochstadt and Lieberman [7]. In [9,10], Gesztesy, Simon and Malamud gave some new uniqueness results in inverse spectral analysis with partial information on the potential for scalar and matrix Sturm-Liouville equations, respectively. Gesztesy and Simon [9] showed that more information on the potential can compensate for less information on the spectrum. In 2001, Sakhnovich [11] studied the existence of solution to the half inverse problem. Recently half inverse problems for Sturm Liouville operators have been studied extensively [12,13].

Some versions of eigenvalue problems for the Sturm-Liouville equation with specified singularities were studied in $[14-23]$, etc. However, for the Sturm-Liouville operator with Coulomb potential, half inverse problems have not been studied yet. The aim of this work is to solve half inverse problem for this operator. This will give the extension of the well-known result in [7].

Before giving the main results, we mention some physical properties of the Sturm-Liouville operator with Coulomb potential. Learning about the motion of electrons moving under Coulomb potential is of significance in quantum theory. Solving these types of problems provides us to find energy levels not only hydrogen atom but also single valance electron atoms such as sodium. For hydrogen atom, Coulomb potential is given by $U=\frac{-e^{2}}{r}$, where $r$ is the radius of the nucleus, $e$ is electronic charge. According to this, we use time dependent Schrödinger equation;

$$
i \hbar \frac{\partial C}{\partial t}=-\frac{\hbar^{2}}{2 m} \frac{\partial^{2} C}{\partial x^{2}}+U(x, y, z) C, \quad \int_{R^{3}}|C|^{2} d x d y d z=1,
$$

where $C$ is the wave function, $\hbar$ is Planck's constant and $m$ is the mass of electron. In this equation, if it is applied Fourier transform

$$
\widetilde{C}=\frac{1}{\sqrt{2 \pi}} \int_{-\infty}^{\infty} e^{-i \lambda t} C d t,
$$

it will convert to energy equation dependent to situation as following;

$$
\frac{\hbar^{2}}{2 m} \nabla^{2} \widetilde{C}+\widetilde{U} \widetilde{C}=E \widetilde{C}
$$

\footnotetext{
* Corresponding author e-mail: murat_sat24@ @otmail.com
} 
Therefore, energy equation in the field with Coulomb potential become as following;

$$
-\frac{\hbar^{2}}{2 m} \nabla^{2} \widetilde{C}+\left(E+\frac{e^{2}}{r}\right) \widetilde{C}=0
$$

If this hydrogen atom is substituted to other potential area, then energy equation become as following;

$$
-\frac{\hbar^{2}}{2 m} \nabla^{2} \widetilde{C}+\left(E+\frac{e^{2}}{r}+q(x, y, z)\right) \widetilde{C}=0 .
$$

If we make the necessary transformation, then we can get a Sturm-Liouville equation with Coulomb potential

$$
-y^{\prime \prime}+\left[\frac{A}{x}+q(x)\right] y=\lambda y
$$

where $\lambda$ is a parameter which corresponds to the energy and $A$ is real constant $[24,25]$.

We consider the singular Sturm-Liouville problem

$$
L y \equiv-y^{\prime \prime}+\left[\frac{A}{x}+q(x)\right] y=\lambda y,(0<x \leq \pi), \quad \lambda=s^{2},
$$

with the boundary conditions

$$
\begin{gathered}
y(0)=0, \\
y^{\prime}(\pi)-H y(\pi)=0,
\end{gathered}
$$

in which the function $q(x) \in L^{1}[0, \pi], H$ is real constant and $\frac{y(x)}{x} \in C[0, \pi]$. The operator $L$ is self adjoint on $L_{2}(0, \pi)$ and with (2), (3) boundary conditions has a discrete spectrum $\left\{\lambda_{n}\right\}$.

\section{Main Results}

Let us denote by

$$
\varphi(x, s)=\sin s x+\int_{0}^{x} \frac{\sin s(x-t)}{s}\left[\frac{A}{t}+q(t)\right] \varphi(t, s) d t
$$

the solution of the equation (1) satisfying the initial conditions

$$
\varphi(0, s)=0 \text { and } \varphi_{x}^{\prime}(0, s)=s,
$$

where $\frac{\varphi(t, s)}{s} \in C[0, \pi]$.

Eigenvalues of the problem (1)-(3) are roots of the (3). In [20] it is shown that these spectral characteristics and and eigenfunctions satisfy the following asymptotic expression, respectively:

$\left.s_{n}=\sqrt{\lambda_{n}}=n+\frac{1}{2}+\frac{A}{2 \pi} \frac{\ln \left(n+\frac{1}{2}\right)}{\left(n+\frac{1}{2}\right)}+\frac{c_{0}}{\left(n+\frac{1}{2}\right)}+O\left(\frac{\ln n}{n^{2}}\right)\right)$,

$$
\begin{aligned}
\varphi\left(x, \lambda_{n}\right)= & \sin \left(n+\frac{1}{2}\right) x+\frac{A}{2 \pi} \frac{\ln \left(n+\frac{1}{2}\right) x}{\left(n+\frac{1}{2}\right)} \cos \left(n+\frac{1}{2}\right) x \\
& +\frac{A \pi}{4} \frac{\sin \left(n+\frac{1}{2}\right) x}{\left(n+\frac{1}{2}\right)}-\frac{\cos \left(n+\frac{1}{2}\right) x}{\left(n+\frac{1}{2}\right)} \beta(x) \\
& -\frac{A}{2} \frac{\cos \left(n+\frac{1}{2}\right) x}{\left(n+\frac{1}{2}\right)} \ln \left(n+\frac{1}{2}\right) x \\
& \left.+O\left(\frac{\ln n}{n^{2}}\right)\right)
\end{aligned}
$$

$$
\begin{aligned}
\varphi_{n}^{\prime}(x)= & \left(n+\frac{1}{2}\right) \cos \left(n+\frac{1}{2}\right) x-\frac{A}{2 \pi} \sin \left(n+\frac{1}{2}\right) x \ln \left(n+\frac{1}{2}\right) x \\
& +\frac{A \pi}{4} \cos \left(n+\frac{1}{2}\right) x+\sin \left(n+\frac{1}{2}\right) x \beta(x) \\
& +\frac{A}{2} \sin \left(n+\frac{1}{2}\right) x \ln \left(n+\frac{1}{2}\right) x \\
& \left.+O\left(\frac{\ln n}{n^{2}}\right)\right),
\end{aligned}
$$

where $\quad c_{0}=\frac{1}{\pi}\left(A M_{1}-H+\frac{A \ln \pi}{2}+\frac{1}{2} \int_{0}^{\pi} q(t) d t\right)$, $\beta(x)=A M_{1}+\frac{1}{2} \int_{0}^{x} q(t) d t, \quad M_{1}=M+\frac{\sin 2}{2} \quad$ and $M=\int_{0}^{1} \frac{\sin ^{2} \xi}{\xi} d \xi$.

Theorem 2.1. We consider the equation (1) with the boundary conditions (2), (3). Let $\left\{\lambda_{n}\right\}$ be the spectrum of the $L$ with conditions (2), (3).

Considering a second operator

$$
\widetilde{L} y \equiv-y^{\prime \prime}+\left[\frac{A}{x}+\widetilde{q}(x)\right] y=\lambda y
$$

where is $\widetilde{q}(x) \in L^{1}[0, \pi]$ and $q(x)=\widetilde{q}(x)$ on $\left[\frac{\pi}{2}, \pi\right]$. Suppose that the spectrum of $\widetilde{L}$ with the conditions (2), (3) is also $\left\{\lambda_{n}\right\}$. Then $q(x)=\widetilde{q}(x)$ almost everywhere on $(0, \pi]$.

Proof. Before proving the theorem, we will mention some results, which will be needed later. Weconsider the initial value problems:

$$
\begin{aligned}
-y^{\prime \prime}+\left[\frac{A}{x}+q(x)\right] y & =\lambda y, \\
y(0) & =0,
\end{aligned}
$$

and

$$
\begin{aligned}
-\widetilde{y}^{\prime \prime}+\left[\frac{A}{x}+\widetilde{q}(x)\right] \widetilde{y} & =\lambda \widetilde{y}, \\
\widetilde{y}(0) & =0 .
\end{aligned}
$$

It can be shown [20] that there exists a kernel $K(x, t)(\widetilde{K}(x, t))$ continuous on $(0, \pi) \times(0, \pi)$ such that by using the transformation operator every solution of equations (10), (11) and (12), (13) can be expressed in the form:

$$
y(x, \lambda)=\sin \sqrt{\lambda} x+\int_{0}^{x} K(x, t) \sin \sqrt{\lambda} t d t+O\left(\frac{e^{\tau x}}{\sqrt{\lambda}}\right),
$$




$$
\widetilde{y}(x, \lambda)=\sin \sqrt{\lambda} x+\int_{0}^{x} \widetilde{K}(x, t) \sin \sqrt{\lambda} t d t+O\left(\frac{e^{\tau x}}{\sqrt{\lambda}}\right),
$$

respectively, where the kernels $K(x, t)(\widetilde{K}(x, t))$ is obtained from the solution of a certain Goursat problem associated with the equation

$$
\left.\frac{\partial^{2} K(x, t)}{\partial x^{2}}-\left[\frac{A}{x}+q(x)\right] K(x, t)=\frac{\partial^{2} K(x, t)}{\partial t^{2}}-\frac{A}{t} K x, t\right)
$$

subject to the boundary conditions

$$
\begin{aligned}
2 \frac{d K(x, x)}{d x} & =q(x), \\
K(x, 0) & =0,
\end{aligned}
$$

where $\tau=|\operatorname{Im} \sqrt{\lambda}|$. This problem can be solved by using Riemann method $[26,27]$.

Using equations (14) and (15), we find that

$$
\begin{aligned}
y(x, \lambda) \widetilde{y}(x, \lambda)= & \sin ^{2} \sqrt{\lambda} x \\
& +\int_{0}^{x}(K(x, t)+\widetilde{K}(x, t)) \sin \sqrt{\lambda} x \sin \sqrt{\lambda} t d t \\
& +\int_{0}^{x} K(x, t) \sin \sqrt{\lambda} t d t \int_{0}^{x} \widetilde{K}(x, m) \sin \sqrt{\lambda} m d m \\
& +O\left(\frac{e^{\tau x}}{\sqrt{\lambda}}\right) .
\end{aligned}
$$

Extending the range of $K(x, t), \widetilde{K}(x, t)$ with respect to the second argument, i.e. $K(x,-t)=K(x, t)$, $\widetilde{K}(x,-t)=\widetilde{K}(x, t)$ and applying some straight forward computation, we find that,

$$
\begin{aligned}
y(x, \lambda) \widetilde{y}(x, \lambda)= & \frac{1}{2}(1-\cos 2 \sqrt{\lambda} x) \\
& +\frac{1}{2} \int_{0}^{x} \widetilde{\widetilde{K}}(x, \xi) \cos 2 \sqrt{\lambda} \xi d \xi \\
& +O\left(\frac{e^{\tau x}}{\sqrt{\lambda}}\right),
\end{aligned}
$$

where

$$
\begin{aligned}
\widetilde{\widetilde{K}}(x, \xi)= & 2[K(x, x-2 \xi)+\widetilde{K}(x, x-2 \xi)] \\
& +2 \int_{-x+2 \xi}^{x} K(x, r) \widetilde{K}(x, r-2 \xi) d r \\
& +2 \int_{-x}^{x-2 \xi} K(x, r) \widetilde{K}(x, r+2 \xi) d r .
\end{aligned}
$$

Next, we define the function

$$
W(\lambda)=y^{\prime}(\pi, \lambda)-H y(\pi, \lambda) .
$$

The zeros of $W(\lambda)$ are the eigenvalues of $L$ or $\widetilde{L}$ subject to conditions (2), (3). The asymptotic results of (7) and (8) imply that $y(x, \lambda)$ and $\tilde{y}(x, \lambda)$ are entire functions of order $\frac{1}{2}$ and hence $W(\lambda)$ is also an entire function of order $\frac{1}{2}$ of $\lambda$.
If we multiply equation (10) by $\widetilde{y}(x, \lambda)$, equation (12) by $y(x, \lambda)$ and then subtract them, we obtain, after integration $[0, \pi]$, we get

$$
\begin{aligned}
{\left[\widetilde{y}(x, \lambda) y^{\prime}(x, \lambda)-y(x, \lambda) \widetilde{y}(x, \lambda)\right] } & \mid \begin{array}{l}
\pi \\
x=0
\end{array} \\
+\int_{0}^{\pi}(\widetilde{q}(x)-q(x)) y(x, \lambda) \widetilde{y}(x, \lambda) d x & =0 .
\end{aligned}
$$

Together with the initial conditions at zero and the assumption $\widetilde{q}(x)=q(x)$ on on $\left[\frac{\pi}{2}, \pi\right]$, then it yields

$$
\begin{aligned}
0= & {\left[\widetilde{y}(\pi, \lambda) y^{\prime}(\pi, \lambda)-y(\pi, \lambda) \tilde{y}^{\prime}(\pi, \lambda)\right] } \\
& +\int_{0}^{\frac{\pi}{2}}(\widetilde{q}(x)-q(x)) y(x, \lambda) \widetilde{y}(x, \lambda) d x .
\end{aligned}
$$

Denote

$$
Q(x)=\widetilde{q}(x)-q(x),
$$

and

$$
H(\lambda)=\int_{0}^{\frac{\pi}{2}} Q(x) y(x, \lambda) \widetilde{y}(x, \lambda) d x .
$$

From the properties of $y(x, \lambda)$ and $\tilde{y}(x, \lambda)$ are considered, the function $H(\lambda)$ is an entire function. For $\lambda=\lambda_{n}$, since the first term of equation (21) is zero, hence

$$
H\left(\lambda_{n}\right)=0, n \in \mathbb{N} .
$$

In addition, using (16) and (23) for $0<x \leq \pi$,

$$
|H(\lambda)| \leq B e^{\tau \pi},
$$

where $B$ is constant and $\tau=|\operatorname{Im} \sqrt{\lambda}|$. By using the asymptotic forms of (7) and (8), we obtain

$$
W(\lambda)=\left(n+\frac{1}{2}\right) \cos \left(n+\frac{1}{2}\right) \pi+O\left(\frac{\ln n}{n}\right) .
$$

Now we define

$$
\Psi(\lambda)=\frac{H(\lambda)}{W(\lambda)}
$$

which is entire function from the above argument and it follows from asymptotic form of $W(\lambda)$ and with equation (25) that we have

$$
|\Psi(\lambda)|=O\left(\frac{1}{\sqrt{\lambda}}\right),
$$

for all $|\lambda|$ enough large. Thus, from the Liouville's theorem, we obtain far all $\lambda$

$$
\Psi(\lambda)=0
$$

or

$$
H(\lambda)=0
$$

Further substituting equation (17) into equation (23), we have

$$
\begin{aligned}
0= & \int_{0}^{\frac{\pi}{2}} Q(x)(1-\cos 2 \sqrt{\lambda} x) d x \\
& +\int_{0}^{\frac{\pi}{2}} Q(x)\left[\int_{0}^{x} \widetilde{\widetilde{K}}(x, \xi) \cos 2 \sqrt{\lambda} \xi d \xi\right] d x \\
& +O\left(\frac{e^{\tau x}}{\sqrt{\lambda}}\right) .
\end{aligned}
$$


This equation can be written as

$$
\begin{aligned}
0= & \int_{0}^{\frac{\pi}{2}} Q(x) d x \\
& -\int_{0}^{\frac{\pi}{2}} \cos 2 \sqrt{\lambda} \xi\left[Q(\xi)+\int_{\xi}^{\frac{\pi}{2}} \widetilde{\widetilde{K}}(x, \xi) Q(x) d x\right] d \xi \\
& +O\left(\frac{e^{\tau x}}{\sqrt{\lambda}}\right) .
\end{aligned}
$$

Letting $\lambda \rightarrow+\infty(\lambda \in \mathbb{R})$, from Riemann-Lebesgue lemma, we obtain

$$
\int_{0}^{\frac{\pi}{2}} Q(x) d x=0
$$

and

$$
\int_{0}^{\frac{\pi}{2}} \cos 2 \sqrt{\lambda} \xi\left[Q(\xi)+\int_{\xi}^{\frac{\pi}{2}} \widetilde{K}(x, \xi) Q(x) d x\right] d \xi=0 .
$$

From the completeness of the function systems $\{\cos 2 \sqrt{\lambda} \xi, \lambda \in \mathbb{R}\}$, we get

$$
Q(\xi)+\int_{\xi}^{\frac{\pi}{2}} \widetilde{\widetilde{K}}(x, \xi) Q(x) d x=0,0<\xi<\frac{\pi}{2} .
$$

Since equation (32) is a homogenous Volterra integral equation, it has only trivial solution. Thus we have obtained our result

$$
Q(x)=\widetilde{q}(x)-q(x)=0, \text { a.e. } x \in(0, \pi] .
$$

This completes the proof.

\section{Acknowledgement}

The author is grateful to the anonymous referee for a careful checking of the details and for helpful comments that improved this paper.

\section{References}

[1] D. Alpay and I. Gohberg, Operator Theory: Advances and Applications, 127, 1-27 (2001).

[2] G. Borg, Acta Mathematica, 78, 1-96 (1946).

[3] F. Gesztesy and B. Simon, Ann. Math., 152, 593-643 (2000).

[4] H. Hochstadt, Comm. Pure Appl. Math., 27, 715-729 (1973).

[5] B. M. Levitan, Mathematics of the USSRIzvestiya, 12, 79193 (1978).

[6] J. Pöschel and E. Trubowitz, Acedemic Press, Orlando, FL., (1987).

[7] H. Hochstadt and B. Lieberman, SIAM Journal of Applied Mathematics, 34, 676-680 (1978).
[8] O. H. Hald, Comm. Pure Applied Mathematics, 37, 539-577 (1984).

[9] F. Gesztesy and B. Simon, Transactions of the American Mathematical Society, 6, 2765-2787 (2000).

[10] M. M. Malamud, Trans. Moscow Math. Soc., 60, 204-262 (1999).

[11] L. Sakhnovich, Inverse Problems, 17, 527-532 (2001).

[12] C-F Yang and Z-Y Huang, Numerical Functional Analysis and Optimization, 31, 754-762 (2010).

[13] Y-P Wang, Result in Mathematics, DOI 10.1007/s0025-0120258-6, (2012).

[14] E. S. Panakhov and M. Sat, doi:10.1186/1687-2770-201349, Boundary value problems, (2013).

[15] E. S. Panakhov and M. Sat, Computer modeling and engineering sciences, 1, 1-11 (2012).

[16] M. Sat and E. S. Panakhov, International Journal of Pure and Applied Mathematics, 80, 173-180 (2012).

[17] V. A. Yurko, Matematicheskie Zametki, 1, 143-156 (1998), English translation in Mathematical Notes, 1, 121-132 (1998).

[18] V. A. Yurko, Differentsial'nye Uravneniya,(Russian), 8, 1355-1362 (1992), English translation in Differential Equations, 28, 1100-1107 (1992).

[19] H. Koyunbakan and E. S. Panakhov, Applicable Analysis, 84, 247-252 (2005).

[20] R. Kh. Amirov, Y. Cakmak and S. Gulyaz, Indian J. Pure Appl. Math., 37, 125-140 (2006).

[21] R. Kh. Amirov and N. Topsakal, Integral Transforms and Special Functions, 19, 923-937 (2008).

[22] R. Carlson, Journal of differential equation, 106, 121-140 (1993).

[23] M. G. Gasymov, DAN SSSR, 161, 271-276 (1965).

[24] D. I. Blohincev, Foundations of Quantum Mechanics, GITTL, Moscow, (1949).

[25] V. A. Fok, Beginnings of Quantum Mechanics, Izdat. Leningrad Gos. Univ., (1932).

[26] R. Courant and D. Hilbert, Methods of Mathematical Physics, New York, (1953).

[27] V. Ya. Volk, Uspekhi Matematicheskikh Nauk, 4, 141-151 (1953).

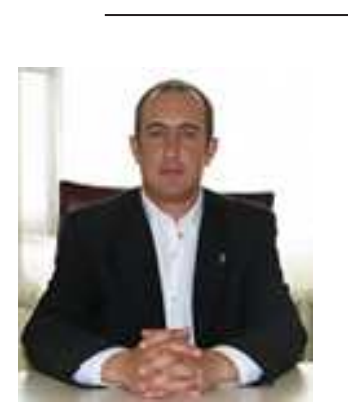

\section{Murat Sat}

$\mathrm{He}$ received his B.S. degree in mathematics (2003) from Cumhuriyet university, M.S. in mathematics (2007) from Ataturk university and Ph.D. degree in mathematics (2012) from Firat university, Elazig, Turkey, in the field of Applied Mathematics. His research interests include spectral theory, inverse problems. He works as an assistance professor of the mathematics department at Erzincan University. 